\title{
Modification of the House of Quality to Assess Information Gaps During Quality Function Deployment of Engineering Design
}

\section{Chelsea Leachman, Washington State University}

Chelsea Leachman is the engineering librarian at Washington State University. She obtained here Masters of Library and Information Science from the University of Wisconsin-Milwaukee in 2011. She has a background in science and engineering. She received her Bachelor of Science in Environmental Science with a minor in geology from the University of Idaho 2007.

\section{Prof. Jacob William Leachman, Washington State University}

Jacob Leachman is an Assistant Professor in the School of Mechanical and Materials Engineering at Washington State University (WSU). He initiated the HYdrogen Properties for Energy Research (HYPER) laboratory at WSU in 2010 with the mission to advance the Technology Readiness Level (TRL) of hydrogen systems. He received a B.S. degree in Mechanical Engineering from the University of Idaho in 2005 and a M.S. degree in 2007. His master's thesis has been adopted as the foundation for hydrogen fueling standards and custody exchange, in addition to winning the Western Association of Graduate Schools Distinguished Thesis Award for 2008. He completed his Ph.D. in the Cryogenic Engineering Laboratory at the University of Wisconsin-Madison in 2010 on the visco-plastic flow of hydrogenic materials for the fueling of fusion energy machines. He has conducted guest research in the Physical and Chemical Properties of Fluids Group at the National Institute of Standards and Technology (NIST), and the Pellet Fueling of Fusion Plasmas Group at Oak Ridge National Laboratory (ORNL). 


\title{
Modification of the House of Quality to Assess Information Gaps during Quality Function Deployment of Engineering Design
}

\begin{abstract}
The Association of College and Research Libraries (ACRL) Framework for Information Literacy for Higher Education established the need to understand the iterative nature of research through posing of increasingly complex questions. Students must have the ability to monitor information gathered and assess for gaps or weaknesses. Students in lower level undergraduate engineering courses are usually unaware of techniques for monitoring information gathered and assessing for gaps in standalone research papers. Moreover, the management of literature research often becomes overwhelming when engineering students encounter team-based design projects that occur over the course of an entire semester. To address this need, we have modified the Quality Function Deployment (QFD) engineering design method to monitor and assess information resources as a natural outcome of the design process. More specifically we have modified the matrix design method known as the House of Quality to associate literature sources with customer determined design constraints and quantify the coverage of the literature review. We introduce this method of identifying literature gaps and weaknesses using QFD in a junior level mechanical engineering systems design course after an initial client interview. Library instruction included a mix of lecture and active learning activities to engage the students with the iterative nature of research. We were thereby able to incorporate information literacy into the preexisting course workflow using the ACRL framework as a guiding document for information literacy.
\end{abstract}

\section{Introduction}

The engineering design process and the frame "Research as Inquiry" from the new ACRL Framework for Information Literacy for Higher Education have many similarities. The main definition of "Research as Inquiry" from the ACRL framework states that "Research is iterative and depends upon asking increasingly complex or new questions whose answers in turn develop additional questions or lines of inquiry in any field."1 Similarly, the definition of the engineering design process as stated by ABET is:

"Engineering design is the process of devising a system, component, or process to meet desired needs. It is a decision-making process (often iterative), in which the basic sciences, mathematics, and the engineering sciences are applied to convert resources optimally to meet these stated needs." 2

Both the ACRL information literacy framework and design process as defined by ABET are iterative. Students must have the ability to monitor the information gathered and assess for gaps or weaknesses during either process. By assessing for gaps in literature the students can reflect on what information they need to gather leading them to develop additional questions. Engineering students are usually unaware of techniques for monitoring information gathered and assessing for gaps in standalone research papers in lower level undergraduate courses and becomes overwhelming when engineering students are introduced to a semester long team-based design project. 
The importance of systemizing the management of design information is vital to the design process but one of the challenges for students is that "sitting down to have a conversation about how to share information and exchange knowledge is probably the least exciting part of a design

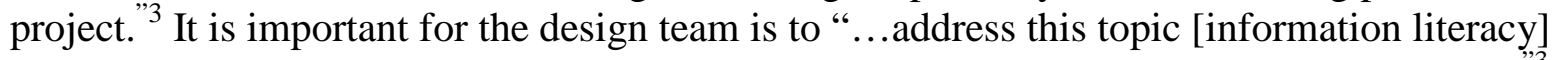
early in the design process to situate the team for maximal efficiency and ultimate success." To address the need for students to engage with information monitoring and understanding the iterative research process, we have modified the matrix design method known as the House of Quality (HOQ) to include associated literature sources with design constraints and quantify the coverage of the literature review.

The HOQ is the core methodology behind Quality Function Deployment (QFD). QFD is a “... means of translating customer requirements into the appropriate technical requirements for each stage of product development and production...." ${ }^{4}$ Originally developed in Japan in 1966, QFD entered the US marketplace in 1983 with a paper published in Quality Progress. ${ }^{5}$ QFD is a heuristic design process that usually follows a four step design progression of 1) product planning, 2) parts deployment, 3) process planning, and 4) production planning, Figure 1.

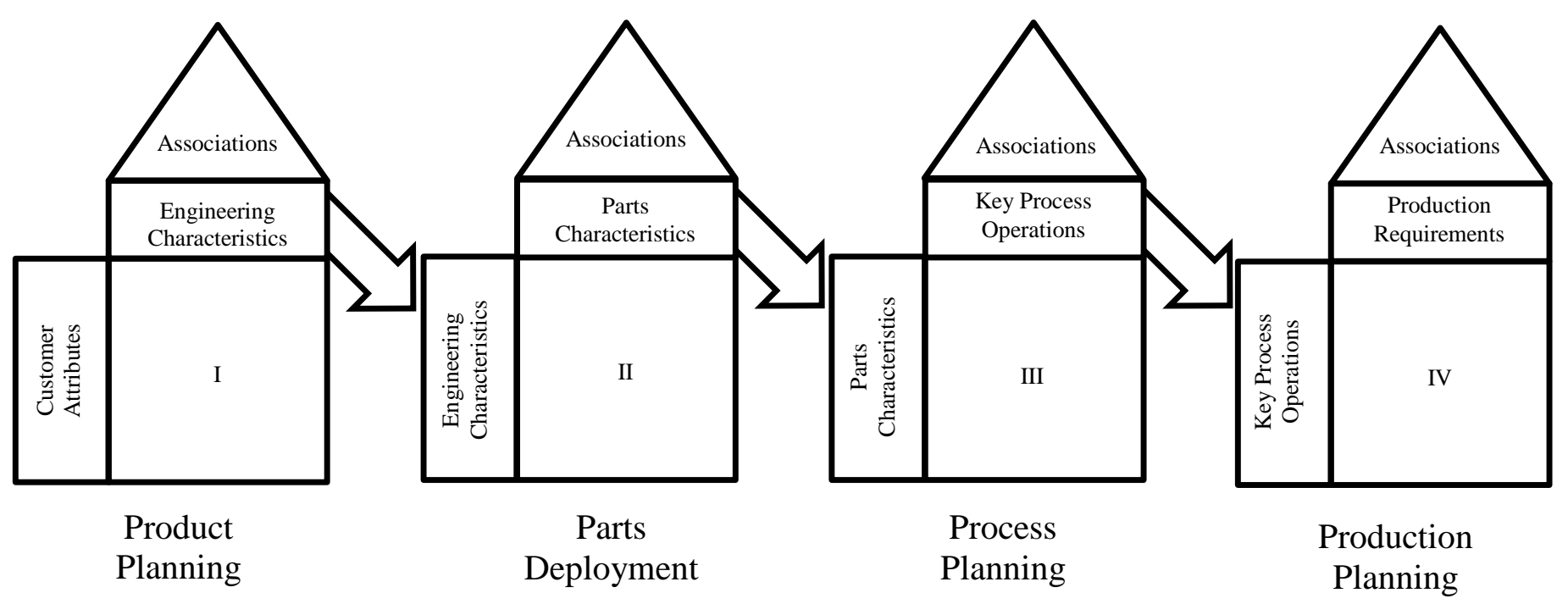

Figure 1: The four design phases of Quality Function Deployment.

The contrasting rows and columns of the HOQ allows relative importance weighting of the customer attributes, multiple associations between attributes, directions for improvement, targets, measureable units, and strengths of associations to be visualized from a single chart. Generally the HOQ is a "... conceptual map that provides the means for interfunctional planning and communications." 6 Figure 2 provides a detailed view of the HOQ showing the "roof" where associations between multiple metrics can be visualized along with the body where weightings are assigned. The "engineering characteristics" in turn become the rows of the parts deployment $\mathrm{HOQ}$, or phase two of QFD, and so on. 


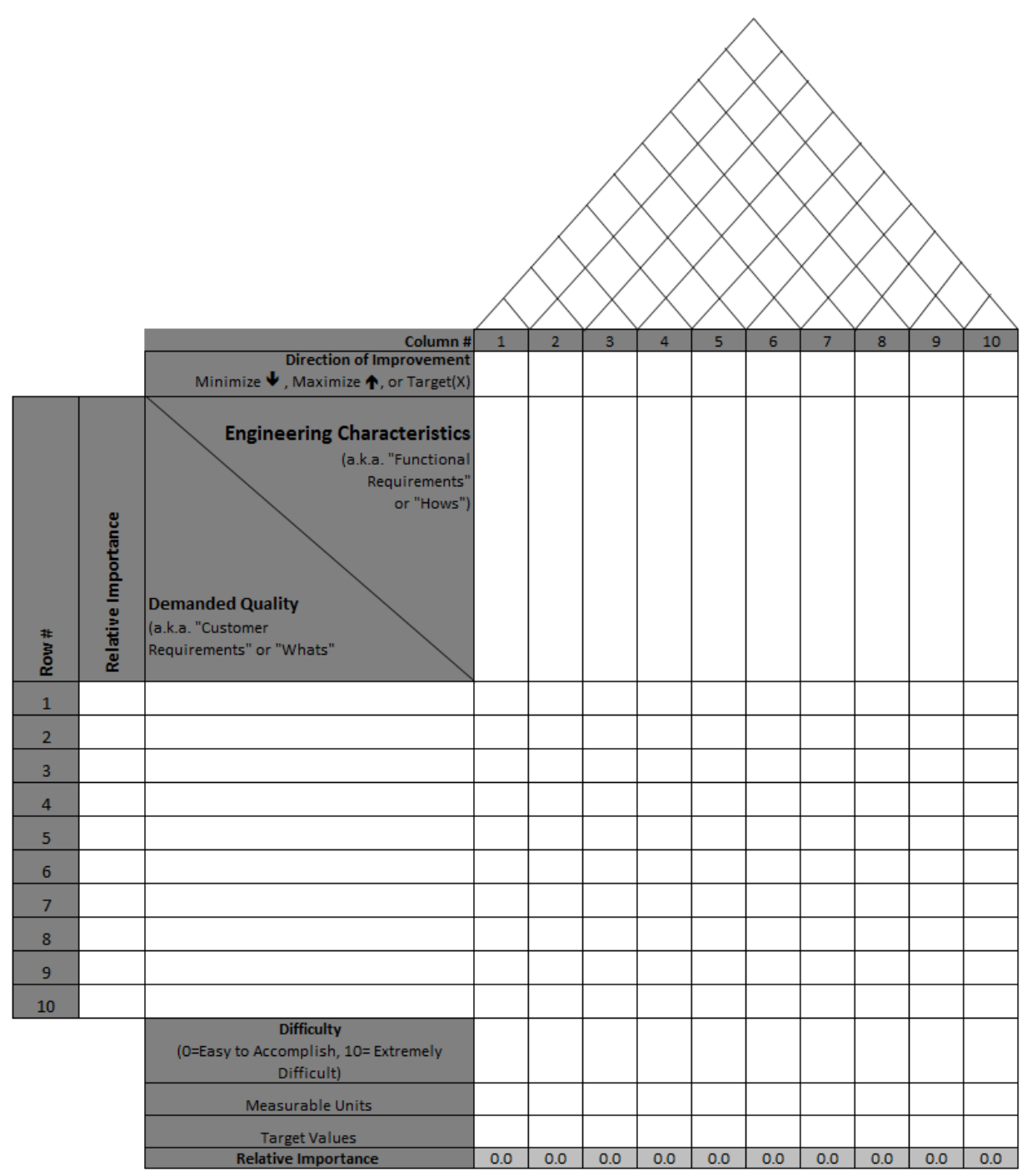

\begin{tabular}{|cl|}
\hline VV & Strong Positive \\
V & Positive \\
- & Negative \\
-- & Strong Negative \\
\hline
\end{tabular}

Figure 2: House of Quality

Traditional instruction methods for completing the HOQ does not include a literature review and assessment as part of the process. Based on informal assessment of students in mechanical engineering, it was evident that methods for incorporating traditional forms of literature into the HOQ are not immediately obvious. After two prior attempts of implementing the HOQ in the design class, students utilized a separate report to list traditional literature forms and did not directly associate these sources with the demanded customer attributes or engineering characteristics of the HOQ. In other words, the students were not systematically identifying gaps in their literature reviews relative to the design requirements. In response to this observation, and the growing need for engineering information literacy, this study was initiated with the following student outcomes:

1. Assess the gaps or weakness of information in the design process in order to select appropriate sources that vary in content, format, and scope.

2. Use technological and organization tools in order to preserve information collected for future use.

3. Utilize an efficient system for gathering, assessing, and storing literature sources relative to design project outcomes. 


\section{Modification of the HOQ}

The closest examples to incorporating a literature review into the HOQ generally come in a "competing technologies" section that is added to the right of the house in the initial product planning phase. These "competing technologies" can then be scored based on the customer attributes and in a sense are a form of "prior art” literature review. However, the "competing technologies" portion of the initial phase of the HOQ is absent in the subsequent parts deployment, process planning, and production planning phases. This "competing technologies" concept can be readily extended to additional phases of QFD to incorporate more traditional literature forms into the HOQ, Figure 3. In addition, this modified HOQ could be used to generate keywords, search methods, systemize the process for finding literature, and identifying key gaps in literature relative to the required engineering characteristics. By including literature sources directly on the HOQ the students visualize the information they already know and see gaps where they still need to gather information. Moreover, collaborative development of the HOQ within design teams allows the team members see what information has already been applied to the design and where new searches or proposed sources of information are needed.

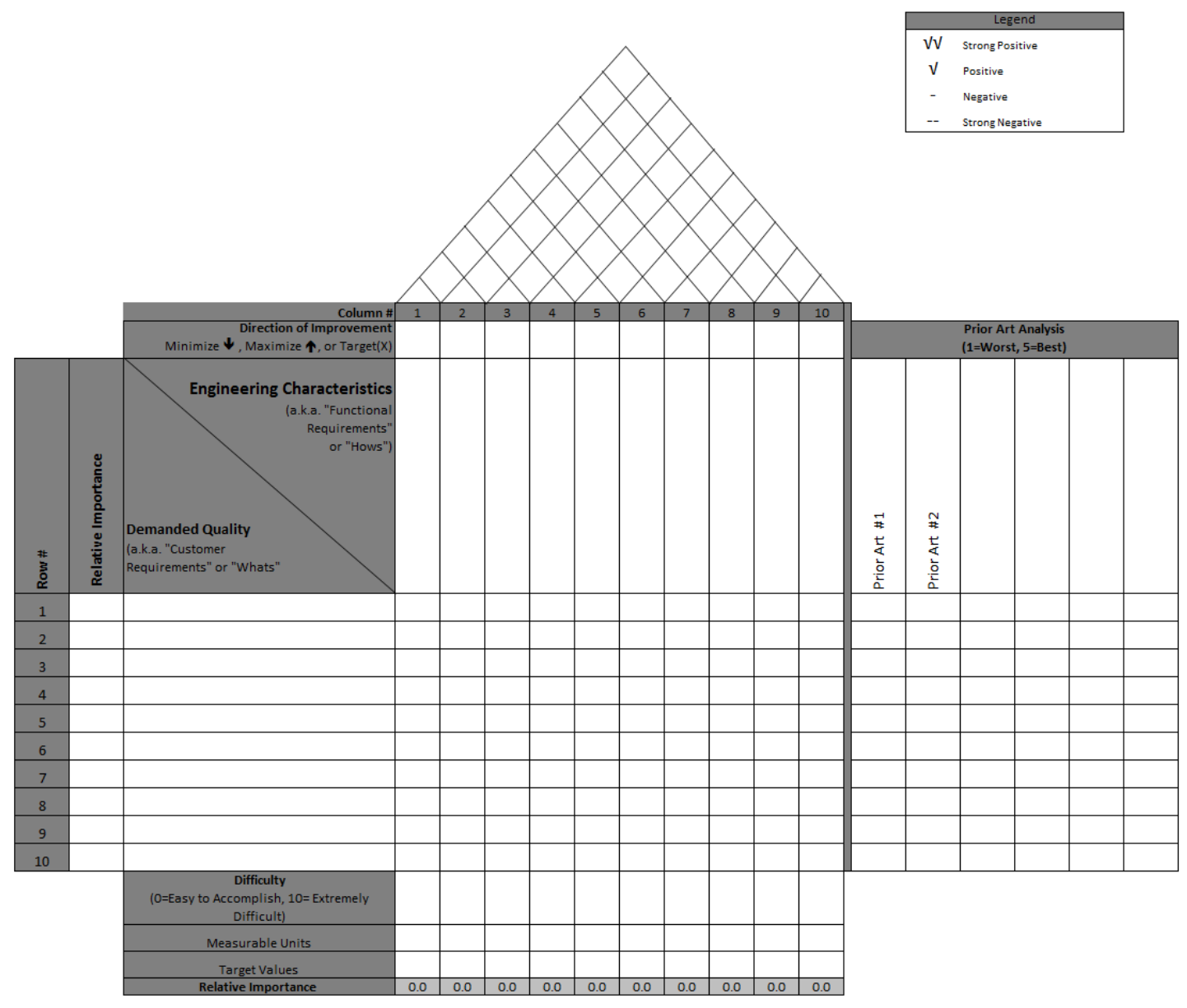

Figure 3: Modified House of Quality Phase 1-3: Modified House of Quality Phase II Parts deployment including a literature analysis section. 


\section{Case Study: Mechanical Systems Design}

A junior level mechanical engineering systems design course was identified for instruction using the modified QFD method for literature tracking. The study was granted an exception to the Institutional Review Board (IRB) human subject testing protocol. The course project was the ambitious undertaking of designing a containerized hydrogen refueling station - a topic the students had little to no prior knowledge with. The 40 students within the course section were divided into eight groups of five students. Each group was responsible for designing a distinctly separate component of the larger fueling station system. As such, significant independent reading of highly technical topics would be required for the teams with this daunting task of systematically sorting the found information.

Library instruction was initiated on the third day of class. The librarian presented an initial overview of library resources and searching methods before focusing on prior art and design standards. Prior to students finding literature sources the librarian lead the class in a source evaluation activity to engage students in thinking about the types and sources of information they would need to find for their design project. During the evaluation activity, student groups were given sources that had been chosen based on their design project to rank on relevance and credibility of the sources. Once the student groups had finished their evaluation of the sources, the librarian led a larger group discussion that transitions students into finding their own sources of information. At the end of class students were asked to engage in information gathering to look for relevant sources to improve vocabulary and terminology in order to complete a stakeholder interview plan prior to meeting the project client. ${ }^{3}$ At this time the QFD was not introduced and the students primary goal was to become more familiar with databases and search terms.

The students interviewed the project client during the fourth day of class. The client is an alumnus of the university that is now the CEO of a company with a committed interest to the design topic. The meeting included a 35-minute presentation of the company's technology and the need for a hydrogen refueling station. The session concluded with a 15-minute unstructured question and answer session.

The course instructor introduced the students to Quality Function Deployment (QFD) and the House of Quality (HOQ) on the fifth day of class. The concept of the QFD and HOQ were covered through an in-class presentation and dissemination of two literature papers on the topic. ${ }^{6,7}$ The students were given the active learning assignment of generating the customer demanded qualities for the HOQ within their design teams. The students then were given the homework assignment of listing engineering characteristics for the entire system to compliment the customer qualities along with identifying prior art.

On the sixth day of instruction, each of the student teams presented their initial HOQ phase I draft to the class. As can be expected, many of the teams interpreted the definition of competing technologies differently and arrays of competitors focusing on different components of the larger system were identified. Typically two to three competing technologies were identified by each team. This process helps the teams to increase awareness of the different aspects of the complex system. The teams were given the homework assignment of providing feedback to three of the 
HOQ's developed by other teams via the Strengths, Improvements, and Insights (SII) model. ${ }^{8}$ The teams were allowed to revise their HOQs before a class vote to select the best HOQ for global adoption. The primary trait of the selected global HOQ included concise listing of differentiable customer demanded qualities and engineering characteristics, Figure 4.

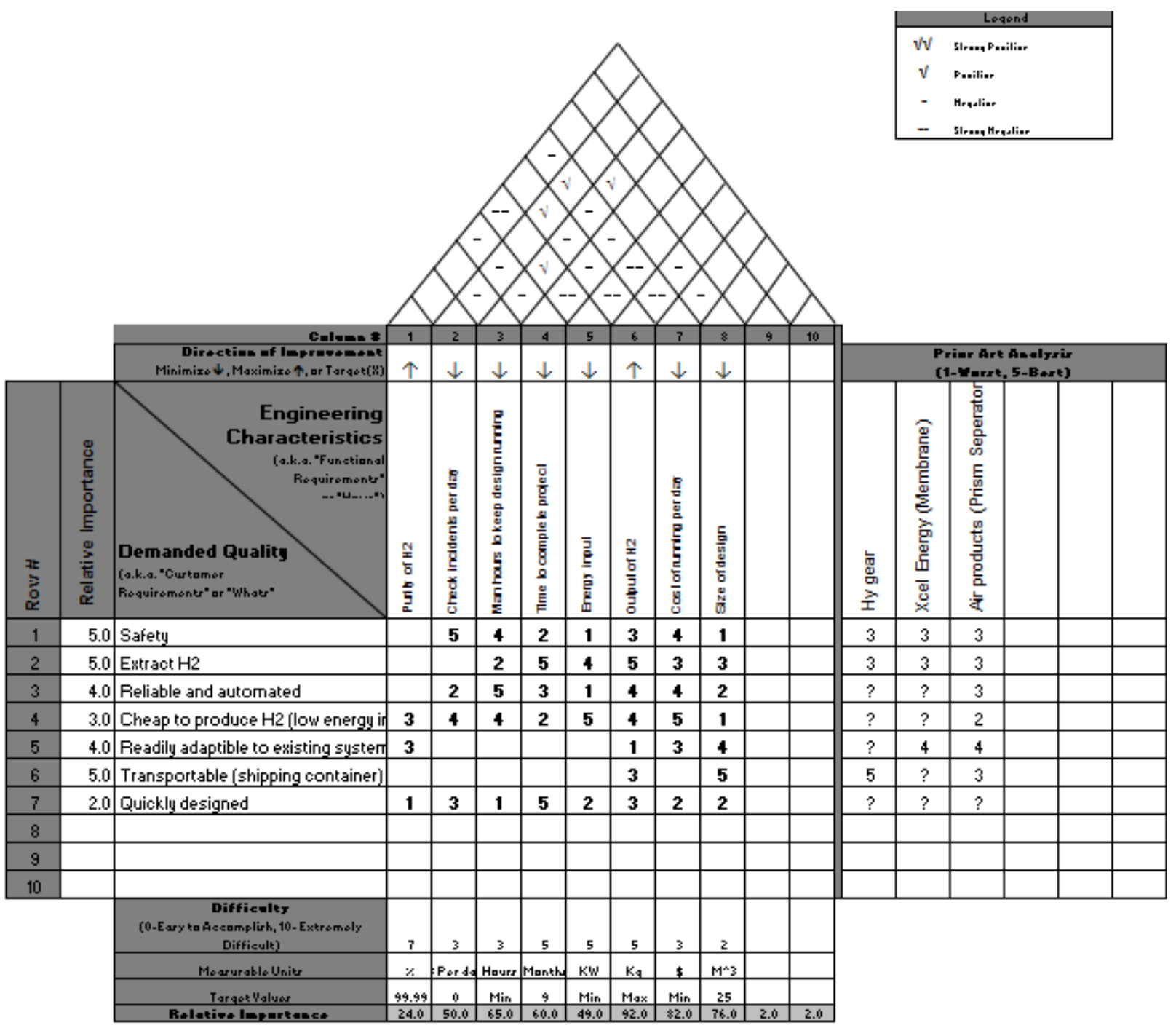

Figure 4: HOQ voted by students to be adopted as global for Phase I.

Adopting a global HOQ for the system based on customer requirements creates a common starting point for each of the teams to assess the performance of their sub-system designs. From the globally adopted HOQ each of the teams moved onto Phase II of QFD by populating the parts characteristics along with also completing a literature review for the engineering characteristics.

Discussion

After working on the parts planning portion of the HOQ over the weekend, teams returned with generally 2-3 sources of relatively moderate credibility. Most of the sources were cited using 
hyperlinks pasted into the literature analysis columns. After observing student work there are opportunities for improving the integration of the literature review into the HOQ.

First, it should be emphasized to students that not all measureable characteristics need a literature component. One example listed by students was “Time to complete project”, while important, does not necessarily require a literature source. While it is important to identify gaps, it's also important to acknowledge that certain gaps are acceptable. This is also an opportunity to revise the validity of the selected engineering characteristics.

Second, the group work reviewed for step two of the QFD included a mix of literature types used to cover the characteristics. While the literature sources were listed, some only had the title or a link to the sources. Although it was mentioned to students that they need to keep track of the literature no specifics were provided. An improvement to the process will include recording their literature sources more formally via adaptation or modification of common citation reference styles. It is indeed fascinating that students instinctively use hyperlinks instead of citations.

Future opportunities for study include the implications of using the HOQ modification on keyword selection. Visualizing the literature analysis alongside the design analysis gives students the ability to identify terminology and search strategies. Additional quantitative assessment of literature sources (e.g., CRAAP test) can be integrated into additional columns or rows of the HOQ.

Overall, integration of the literature review into the QFD system via the HOQ encouraged students to think about the literature they were using and assessing for literature gaps relative to the customer demanded qualities. The process of posing increasingly complex questions is natural in the design process and through integrating the literature review process the students were able to grasp large amounts of information. The process of using the HOQ does require instructor buy-in and coordination over the course of a few weeks, which could be a limitation in some situations.

References

1. Association of College and Research Libraries, "Framework for Information Literacy for Higher Education", Chicago, Illinois: American Library Association, 2015.

2. Engineering Accreditation Commission," Criteria for accrediting engineering programs", ABET Report E001 11/7/14, 2014.

3. Radcliffe, D., and M. Fosmire, Integrating Information Into the Engineering Design Process: Purdue University Press, 2014.

4. $\quad$ Sullivan, L.P.," Quality Function Deployment", Quality Progress Vol. 19, No. 6, 1986, pp. 39-50.

5. $\quad$ Chan, L.-K., and M.-L. Wu," Quality function deployment: A literature review", European Journal of Operational Research Vol. 143, No. 3, 2002, pp. 463-497.

6. Hauser, J.R., and D. Clausing, "The house of quality", Harvard Business Review Vol. 66, No. 3, 1988, pp. 63-74.

7. Govers, C.P.M., "What and how about quality function deployment (QFD)”, Int. J. Production Economics Vol. 46-47, 1993, pp. 575-585. 
8. Parker, P.E., P.D. Fleming, S. Beyerlein, D. Apple, and K. Krumsieg, "Differentiating assessment from evaluation as continuous improvement tools," $31^{\text {st }}$ ASEE/IEEE Frontiers in Education Conference, October 10-13, Reno, NV, 2001. 